\title{
Self-Defense: A Practical Approach to Combatting COVID-19
}

\author{
Salam A Ibrahim ${ }^{1 *}$, Rabin Gyawali ${ }^{1}$ and Hafize Fidan ${ }^{2}$ \\ ${ }^{1}$ Food Microbiology and Biotechnology Laboratory, North Carolina Agricultural and \\ Technical State University, Greensboro, NC, USA \\ ${ }^{2}$ Department of Nutrition and Tourism, University of Food Technologies, Plovdiv, \\ Bulgaria \\ *Corresponding Author: Salam A Ibrahim, Food Microbiology and Biotechnology \\ Laboratory, North Carolina Agricultural and Technical State University, \\ Greensboro, NC, USA.
}

Received: May 11, 2020

Published: June 08, 2020

(C) All rights are reserved by Salam A

Ibrahim., et al.

\section{Abstract}

In order to elucidate the importance of the immune system with regard to the spread, diagnosis, and treatment of the novel COVID-19, this paper aims to emphasize the need for a more holistic approach to understand and treat the COVID-19. We have provided an overview of why high quality nutrition is important for the immune system to function properly. To do this, we have summarized the current evidence available from information being reported by the health authorities and studies in human subjects, to support this opinion. Though the available data in the literature is relatively scarce, we also offer our own informed opinions on the role of human microbiota (probiotics) in creating a natural immunological defense system for combating COVID-19. We thus hope that this paper will help to inspire scientists from other fields such as nutrition immunology to be involved in order to provide consumers with a more global approach to fighting this epidemic. If we want to eliminate the threat of this novel coronavirus pneumonia, we must practically address this issue itself.

Keywords: Self-Defense; SARS-CoV-2; Nutrition; Human Microbiota; Practical Approach

\section{Introduction}

Coronaviruses are a large group of viruses that belong to the family Coronaviridae, subfamily Orthocoronavirinae. These viruses find suitable environments for development and metabolic activity in humans, bats, pigs, cats, dogs, rodents and poultry (domestic and wild animals). It is known that the disease spectrum caused by coronaviruses in humans is more acute than that of the common cold and that the respiratory response syndrome of this virus can vary greatly in different individuals. For example, coronaviruses can cause various respiratory, enteral, liver, nephrotic and neurological diseases in humans and animals [1].

On December 31, 2019, the World Health Organization (WHO) reported cases of pneumonia of unknown etiology in Wuhan, Hu- bei Province, the People's Republic of China (PRC). Subsequently, a new coronavirus (2019-nCoV) that had never before been detected in humans was identified as the causative agent of the respiratory symptoms. Later, this particular coronavirus was renamed SARSCoV-2. The disease subsequently spread rapidly due to person to person transmission [2-4] through China's trade and tourism links into Europe, Asia, and, eventually, North America and the United States (Figure 1).

The disease caused by the novel SARS-CoV-2 is transmitted mainly via the air-dropletsthat results from sneezing and coughing or after contact with an infected individual. The virus can also be transmitted after touching an infected surface or by contacting with a contaminant when one's hands touch the mouth, nose or eye 


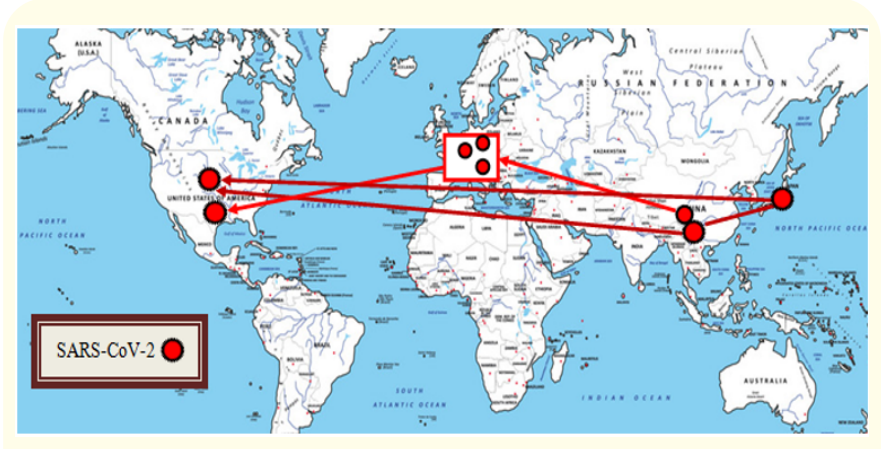

Figure 1: Transmission map of SARS-CoV-2 around the world.

mucosa. SARS-CoV-2, now commonly referred to as COVID-19, can be found in the respiratory secretions of asymptomatic individuals, but the primary mode of transmission is through infected individuals. It is known that the incubation period for COVID-19 can last up to 14 days without knowing the exact duration of infection. Common symptoms of infection include respiratory symptoms, fever, cough, and shortness of breath. In more serious cases, it is known that pneumonia, severe acute respiratory infection, kidney failure, and even death can occur. There is currently no specific treatment with proven safety and efficacy for COVID-19. In order to find an effective treatment for this disease, numerous studies are being carried out, and results are expected to be announced as each study is completed [5].

The World Health Organization has published several recommendations that would prevent the spread and contamination of COVID-19, such as: avoiding contact with infected or potentially infected people (at least keeping $1 \mathrm{~m}$ physical distancing ), adhering to food safety recommendations (refrain from consuming raw milk and animal feed products), proper washing of fruits and vegetables that are consumed raw, avoiding contact with wild and domestic animals (living or dead), observing prudent hygiene measures e.g. constant washing and disinfecting hands; keeping an appropriate social distance and social exclusion; wearing masks; using disposable wipes and for sneezing and coughing, using the inside of the elbow if a disposable tissue or cloth is not available [6].

According to a WHO report regarding China, fatalities caused by COVID-19 more typically occur in the elderly who have other accompanying disorders such as hypertension, diabetes, cardiovascular disease, cancer, chronic lung disease or other immunosuppressive conditions. Several measures and practices have thus been proposed to reduce if not eliminate the spread of this novel virus including stay-at-home orders of self- isolation, good personal hygiene and practices, maintaining social distancing, the use of masks and avoiding social gatherings, practicing respiratory hygiene, and avoiding touching the eyes, nose, and mouth [3].

However, with the spread of this virus and its respiratory disease to 212 countries around the world, many young people have also been impacted. For example, the lower age limit of potential fatality has been reduced to include 18 year-olds, sometimes without declared concomitant diseases. This is the question that engages the minds of many medics and scientists around the world - how does one individual survive illness at the age of 93, while another at 18 years old does not? The reason for this disparity could be due to a variety of assumptions such as the susceptibility of the body, presence of accompanying diseases, level of entry of viral particles in the body, compliance with hygiene measures in everyday life and many other factors, including the maintenance of the body's defense mechanisms that are assumed to play a significant role in the recovery prospects of the disease.

An individual's immune system is typically the most critical part of the body's defenses against the novel coronavirus. Public health and hygiene practices such as proper handwashing can also help to reduce the impact of COVID-19. However, the development of a safe, effective vaccine could take anywhere from an estimated earliest arrival of 12 months to several years to create, and people from different geographical regions could have variable and unpredictable responses to it. Until a vaccine is available in the global market, our immune systems need to fight against COVID-19. Moreover, if the virus begins to mutate over time, a vaccine could prove to be less and less effective in providing appropriate levels of protection. Thus, the current COVID-19 mortality numbers highlight the need for additional strategies and measures to support the immune system such as enhanced - "self-defense." If we maintain a healthy lifestyle, the immune system's resources can easily tackle invaders and prevent them from entering the body. Proper nutrition also plays an essential role in supporting an individual's self-defense system. 
For example, several vitamins and food groups support both innate and adaptive immune function protecting our body against invaders. Conversely, a deficiency or suboptimal status of macro and micronutrients adversely affects immune functions and may reduce resistance to infection. In this review, we will discuss a practical intervention approach to COVID-19 that we are calling 'self-defense' that can be achieved through the improvement of the immune system.

\section{Immune system}

The human body provides a favorable environment for the development and metabolic activity of many microorganisms including pathogenic bacteria, viruses, and fungi that attempt to enter the body in a variety of ways. These invaders act on the immune system, a collection of cells, tissues, and organs that protect the body from the attacks of foreign organisms that can cause disease. Infections are the most common diseases that range from the common cold to life-threatening diseases such as AIDS. The skin is known to be the body's largest barrier organ, whose primary function is to protect the body from mechanical engagement with the environment and pathogen invasion. Disease-causing microorganisms attempt to invade the host organism through several pathways including the skin (directly or through wounds), the respiratory tract, or the digestive tract. The immune system defends against these pathogenic microorganisms by way of very complex systems. The purpose of the immune system is to distinguish the body's own cells from those that are foreign and pathogenic. The immune system includes lymphoid or small white blood cells that monitor the invasion of foreign cells into the body by "walking" through blood vessels or the lymphatic system. By its origin, the immune system could be innate or acquired. The innate immunity is formed during the embryonic development of an individual, which is related to the genetic characteristics of individuals and the immunity of the maternal organism. The acquired immunity could be active and passive. The naturally active acquired immunity is developed as a result of diseases such as measles, scarlet fever, etc. and the artificial active immunity is acquired after immunization. The natural passive immunity is acquired in the first months after birth when the baby gains antibodies from the mother and artificial passive immunity, when ready-made antibodies are transmitted via hyperimmune serum [7].

\section{The importance of nutrition in the immune system}

The importance of nutrition in enhancing the body's protective functions against a variety of diseases such as polio, diphtheria, tetanus, variola, and others, has been known to people for many decades. In traditional medicine, fruits, herbs, and spices that are included in foods, teas, syrups have been known to cure certain diseases [8]. As food scientists who are interested in practicing the concept of healthy foods for healthy livings, we would like the scientific community to consider the concept of self-defense (selfprotection by enhancing the immune system) of the body as a valid prophylaxis in the era of COVID-19. A good, nutritious diet has long-term benefits for immunity as it is well known that a healthy diet can benefit the immune system and enhance the body's ability to deal with outside threats. Malnutrition breaks down immune functions by suppressing the immune system. Among the factors that cause a detrimental effect on the body's immune function are the inadequate intake of macronutrients (fats, carbohydrates, and proteins), micronutrients (vitamins and minerals) and water (Figure 2). A balanced diet, especially with regard to adequate intake of vitamins, minerals, and proteins, increases resistance to infections. Malnutrition causes the immune system to collapse. Consequently, it is necessary to consume adequate amounts of foods that include protein sources, carbohydrates, and fats, as well as antioxidants such as vitamin $\mathrm{C}, \mathrm{E}$ and foods consisting of beta-carotene and others that can protect the body against free radicals. Scientists do not yet know exactly what happens when a coronavirus infection takes place, but it could be the individual's immune response that is crucial in many cases. Clearly, the virus matters, but the host response is probably more important with regard to disease outcomes and could likewise play a major factor in the spread and treatment of this infectious disease [9].

The importance of human microbiota in the immune system

As we know, personal microbiota plays an important role in human health. The gut microbiome is acquired and varies according to multiple environmental impacts, including geography, diet, and lifestyle. It is likely that improvements in sanitation, food and water in more recent times were involved in reducing our exposures to these microbes [10]. The western world's world lifestyle of excessive cleanliness, clean water and food, sanitation, antibiotics and 


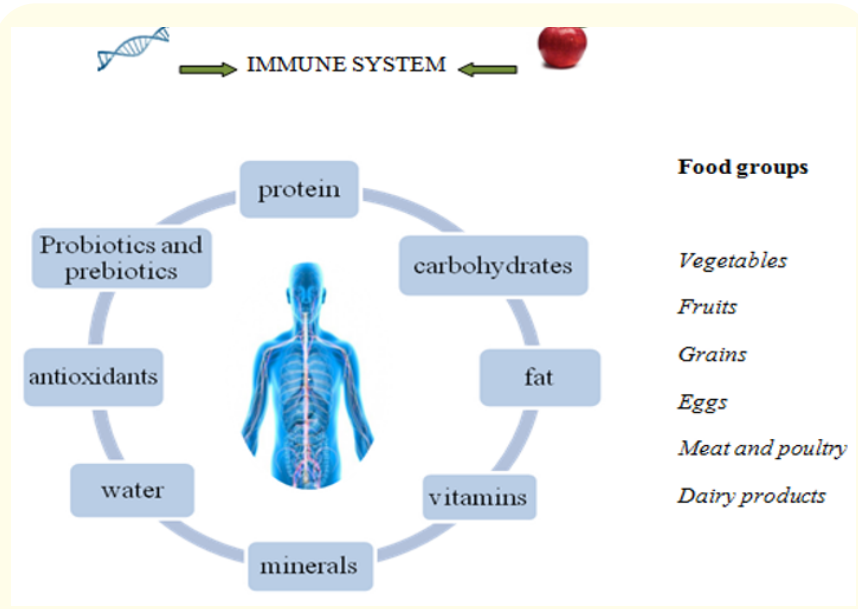

Figure 2: Feeding human's immune system.

vaccines, birth practices, as well as urban and indoor lifestyles could have led to less exposure to these microbe populations. It seems that childhood exposure to microbiota helps the immune system develop. In fact, many children in the western world lack exposure to bacteria, viruses, and allergens, and it is this lack of exposure that ironically prevents the normal development of the immune system, ultimately increasing the chances of immune deficiency diseases. This helps to explain why allergy and asthma rates are so much higher in western countries [11]. Thus, declining microbial exposure is considered as a major causative factor in the increasing incidence of such illnesses. For example, with the recent COVID-19 outbreak, the fatality rates are lower among developing countries compared to developed ones such as Italy, France, Spain, and the United States [12]. We can relate this result to the lifestyle of the people in developing nations where people are more likely to be exposed to microbiota in their day to day life, and household sanitation practices are not as rigid as those in the West. Without being exposed to enough microbiota, our immune systems may not be able to learn to properly recognize harmful invaders. As reported earlier, diet also plays a major role in the diversity of microbiota. Unfortunately, in western and developed nations, people mainly rely on processed foods and less fiber-rich foods. Fiber-rich foods act as a prebiotic by creating a spawning ground for beneficial microbiota [13]. Thus, adherence to a more plant-based diet is warranted. Microbiota is critical to our health, and these microorganisms could be the key to preventing a host of diseases by playing a vital role in the body's self-defense mechanism

\section{Recommendations and Conclusion}

While it is clearly crucial to continue to maintain personal hygiene standards like washing our hands frequently with soap and water, wearing a mask (covering nose \& mouth), covering the mouth while sneezing and coughing, avoiding touching our hands or mouth and using alcohol sanitizers, there are also critical strategies for improving our immune function during this pandemic. In order to maintain a healthy immune system and to fight against viral infection, we need to support our mechanisms of self-defense through a balanced macro diet (proteins, carbohydrates, and fats) and to get adequate amounts of vitamins, minerals, fiber and antioxidants, and other bioactive ingredients of foods and herbs [1416]. For this, the consumption of a well-balanced and healthy diet should be part of everyone's meal in this global crisis. It is important to take steps now to boost our immune system so that the body can effectively fight back when it does come in contact with COVID-19. At the same time, we should not ignore the important role of microbiota in the immune system. However, the use of probiotics and the effectiveness of human microbiota both warrant thorough and extensive research. Based on the current scenario, our understanding of exactly how and why some people get so sick while others feel almost nothing will be the subject of further study.

To sum up, this paper has revealed the importance of the immune system with regard to the spread, diagnosis, and treatment of the novel coronavirus, COVID-19. The paper was created following WHO recommendations as the rapid spread of the virus worldwide has shaped the need to seek new approaches to combat it. In addition to the development of urgently needed vaccines and therapeutic drugs, scientists must also focus on the implementation of a self-defense approach. Consumers are looking to protect themselves and their immune systems by adopting healthier diets. Thus, we sought to open a discussion about the important role of nutrients in enhancing the body's immune response to infections, particularly COVID-19. As a result, we have focused the spotlight squarely on the term self-defense as a valid first line of protection. As the field of medicine is moving toward personalized medicine, the self-defense approach should also be included as a practical approach in addressing the issue of COVID-19.

\section{Acknowledgements}

This publication was made possible by grant number NC.X-2675-12-170-1 from the National Institute of Food and Agriculture 
(NIFA) and the Agriculture Research Station at North Carolina Agriculture and Technical State University (Greensboro, NC, USA).

\section{Conflict of Interest}

The authors declare no conflict of interest.

\section{Bibliography}

1. Cui J., et al. "Origin and evolution of pathogenic coronaviruses”. Nature Reviews Microbiology 17 (2019) 181-192.

2. Deng SQ and Peng HJ. "Characteristics of and Public Health Responses to the Coronavirus Disease 2019 Outbreak in China". Journal of Clinical Medicine 9.2 (2020): 575.

3. World Health Organization. "Report of the WHO-China Joint Mission on Coronavirus Disease 2019 (COVID-19)” (2020).

4. World Health Organization. "Naming the coronavirus disease (COVID-19) and the virus that causes it" (2020).

5. Adamson CS. "Antiviral Agents: Discovery to Resistance". Viruses 12.4 (2020): 406.

6. World Health Organization. "Coronavirus disease (COVID-19) advice for the public: Advocacy" (2020).

7. U.S. Department of Health and Human Services National Institutes of Health. Understanding the Immune System How It Works. National Institute of Allergy and Infectious Diseases National Cancer Institute, NIH Publication No. 03-5423 (2003).

8. Ali HI., et al. "The Effect of nutrition on immune system Review paper". Food Science and Quality Management (2019): 90.

9. Childs CE., et al. "Diet and Immune Function". Nutrients 11.8 (2019): 1933.

10. Scudellari M. "News Feature: Cleaning up the hygiene hypothesis". Proceedings of the National Academy of Sciences 114.7 (2017): 1433-1436.

11. Prescott SL., et al. "A global survey of changing patterns of food allergy burden in children". World Allergy Organization Journal 6.1 (2013): 21.

12. WHO. Coronavirus (COVID-19) (2020).
13. Gyawali R and Ibrahim SA. "Impact of plant derivatives on the growth of foodborne pathogens and the functionality of probiotics". Applied Microbiology and Biotechnology 95.1 (2012): $29-45$.

14. Calder PC., et al. "Optimal nutritional status for a well-functioning immune system is an important factor to protect against viral infections". Nutrients 12.4 (2020): 1181.

15. Galanakis CM. "The Food Systems in the Era of the Coronavirus (COVID-19) Pandemic Crisis". Foods 9.4 (2020): 523.

16. Gombart AF, et al. "A review of micronutrients and the immune system-working in harmony to reduce the risk of infection". Nutrients 12.4 (2020): 236.

\section{Assets from publication with us}

- Prompt Acknowledgement after receiving the article

- Thorough Double blinded peer review

- Rapid Publication

- Issue of Publication Certificate

- High visibility of your Published work

Website: $\underline{w w w}$.actascientific.com/

Submit Article: www.actascientific.com/submission.php

Email us: editor@actascientific.com

Contact us: +919182824667 